\title{
AGILE hunts gravitational waves counterparts
}

\author{
Carlotta Pittori*广 \\ INAF-OAR and ASI Space Science Data Center (previously ASDC), Roma, Italy \\ E-mail: carlotta.pittoriessdc.asi.it
}

\section{Marco Tavani}

INAF-IAPS and Univ. di Roma "Tor Vergata”, Roma, Italy

\begin{abstract}
After the breakthrough discovery of gravitational waves by the LIGO-Virgo collaboration in 2015, the quest for electromagnetic counterparts to extreme gravitational events is now open. We report the results of an extensive search through AGILE data for a high-energy counterpart to the first gravitational wave event ever detected: GW150914. The Italian Space Agency satellite AGILE devoted to gamma-ray astrophysics is operating nominally since its launch on April 23, 2007, and it is continuosly monitoring the sky in a spinning observation mode. With the potential of covering $\sim 80 \%$ of the entire sky with its gamma-ray instrument more than 100 times a day, AGILE is currently at the forefront in the hunt for electromagnetic counterparts of gravitational waves. Even though a gamma-ray counterpart of the GW150914 event was not detected, the prospects for future AGILE observations of GW sources are shown to be very promising.
\end{abstract}

Frontier Research in Astrophysics - II

23-28 May 2016

Mondello (Palermo), Italy

*Speaker.

${ }^{\dagger}$ on behalf of the AGILE Collaboration. 


\section{Introduction}

\section{The AGILE Mission status}

AGILE [1] is an Italian Space Agency (ASI) small class scientific mission for high-energy astrophysics launched on April 23, 2007 in an equatorial orbit with initial altitude of about $550 \mathrm{~km}$. The payload is a small cube of $\sim 60 \mathrm{~cm}$ size, which achieves an effective area of approximately $500 \mathrm{~cm}^{2}$ at several hundreds $\mathrm{MeV}$, an angular resolution of about $4.3^{\circ}$ at $100 \mathrm{MeV}$ (at $68 \%$ containment radius), decreasing below $1^{\circ}$ for energies above $1 \mathrm{GeV}$, and an unprecedentedly large field of view $(\mathrm{FoV})$ of about $\sim 2.5 \mathrm{sr}$. The satellite is working nominally after 10 years of operations, and it is continuously monitoring the sky searching for steady and transient gamma-ray sources in the energy range $30 \mathrm{MeV}-50 \mathrm{GeV}$.

Since Nov. 2009 AGILE is operated in the so called spinning observation mode, with the satellite performing a complete rotation around the Sun-satellite versor roughly every 7 minutes. In this operation mode, the AGILE gamma-ray imager (GRID) can observe approximately $80 \%$ of the sky more than 100 times a day ${ }^{1}$, with a sensitivity to fluxes of the order of $10^{-6} \mathrm{ph} \mathrm{cm}^{-2} \mathrm{~s}^{-1}$ above $30 \mathrm{MeV}$ for typical $\sim 100$ seconds single pass over unocculted sky regions. In addition the AGILE $4 \pi$ non-imaging Mini-Calorimeter (MCAL) detector, working in the range $0.350-100$ $\mathrm{MeV}$, and the anticoincidence (AC) system are routinely employed for the detection of GRBs and TGF, and are capable of detecting gamma-ray transients and GRB-like phenomena for timescales ranging from sub-milliseconds to ten-hundreds of seconds.

\section{AGILE and GW150914}

GW150914 occurred on September 14, 2015 at time T0 = 09:50:45 UTC, and it was the first direct gravitational wave (GW) event ever detected [2]. The AGILE Team was not part of the multifrequency follow-up collaboration with the LIGO-VIRGO Collaboration at the time of the GW150914 detection and subsequent investigations. At that time AGILE was scanning the sky in spinning mode with the Earth only partially occulting the GRID FoV. An extensive archival search through AGILE data for a high-energy counterpart to GW150914 was performed retroactively as soon as the event was made public by the LIGO-VIRGO Collaboration on February 11, 2016.

AGILE FoV was covering a good fraction of the GW150914 localization region at interesting time intervals that preceded and followed the prompt event, and Figure 1 shows the AGILE gammaray exposure above $50 \mathrm{MeV}$ during the 7-minute revolution that includes the GW150914 event time T0. The AGILE-GRID FoV radius is set to $70^{\circ}$, and the exclusion region for the albedo photons is taken to be $80^{\circ}$ from the Earth center. Most of the GW localization region (LALinference $90 \%$ contour level [3, 4]), marked by the purple line in Figure 1, was not occulted by the Earth, and therefore available for AGILE exposure.

We performed a search for prompt emission involving the GRID and MCAL instruments, and $\mathrm{AC}$ and Super-AGILE ratemeters on short time scales ranging from a few to tens of seconds around

\footnotetext{
${ }^{1}$ The total number of 7-minute rotations is $\sim 200 /$ day, but the number of useful gamma-ray exposures is affected by Earth occultations and SAA passages.
} 


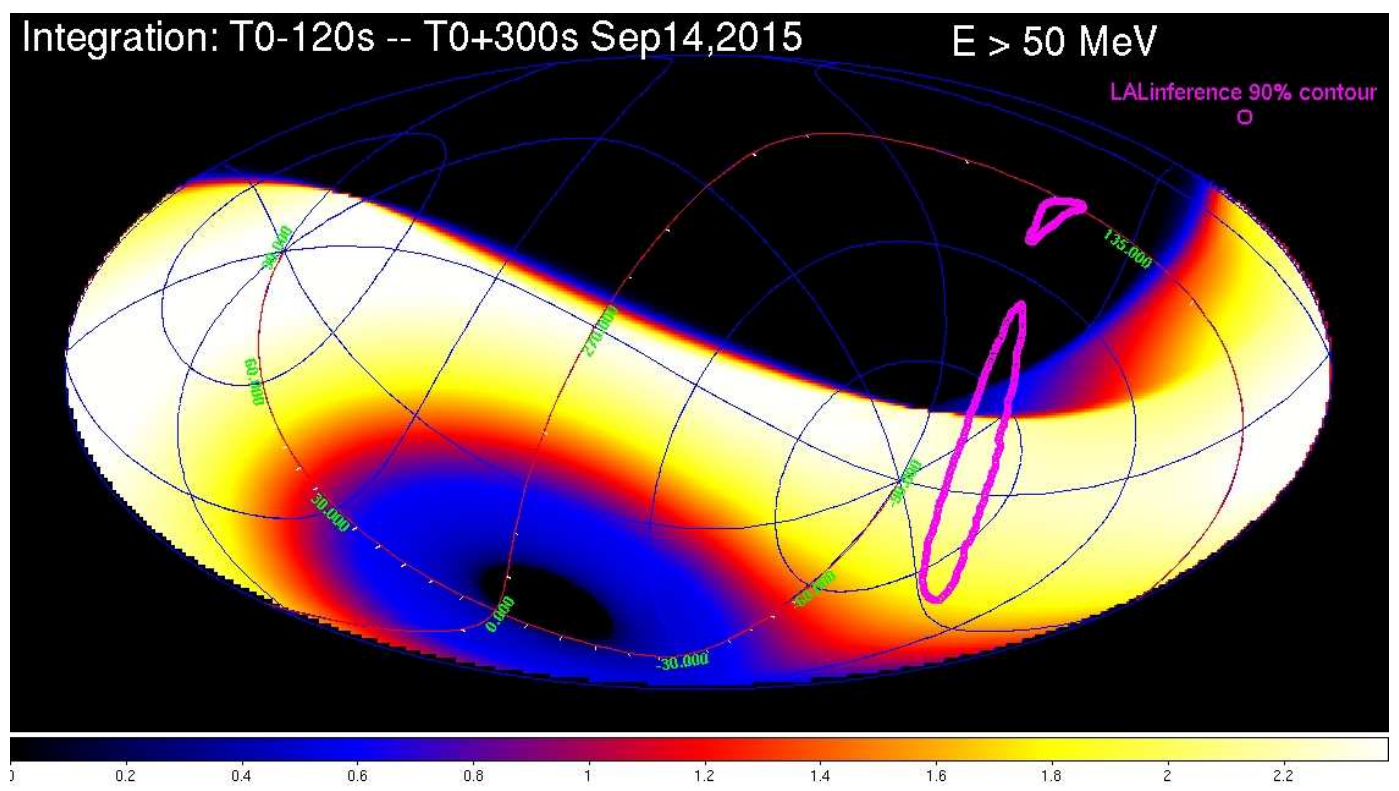

Figure 1: AGILE-GRID cumulative gamma-ray exposure above $50 \mathrm{MeV}$ (in $\mathrm{cm}^{2} \mathrm{~s}$ sr) during the satellite 7-minutes revolution that includes the GW150914 event time. Hammer-Aitoff projection in Galactic coordinates, with equatorial coordinate grid overlay. In this case, the Earth is occulting the GRID FoV only in the Northern Galactic hemisphere. The GW150914 localization region (LALinference 90\% contour level [3, 4]) is marked by the area inside the purple line.

T0, and we also searched for a possible gamma-ray precursor and delayed emission involving the GRID [5].

\subsection{Prompt event}

AGILE-GRID just missed covering the GW150914 localization region during the prompt event with useful sensitivity. AGILE-GRID had exposure of a substantial fraction (65\%) of the GW150914 localization region a few tens of seconds before T0, but not during the $4 \mathrm{sec}$ interval that includes the GW150914 event time, as shown in Figure 2. However a large fraction of the GW localization region was not occulted by the Earth and a strong X/gamma-ray signal, if any, could have been detected by the AGILE non-imaging $4 \pi$ detectors MCAL, AC and Super-AGILE ratemeters.

The MCAL did not register any event above the trigger threshold in the energy range 0.4-100 $\mathrm{MeV}$ within the time interval covering $\pm 100 \mathrm{~s}$ from T0. Also the AC system and Super-AGILE detectors in the ranges $80-200 \mathrm{keV}$ and $20-60 \mathrm{keV}$ respectively, did not register enhancements of their count rates during the prompt event.

In particular, AGILE did not detect the weak candidate transient reported by Fermi-GBM about 0.4 seconds after $T_{0}$ [6]. The Fermi-GBM candidate event position was viewed by AGILEMCAL at large off-axis angles between $90^{\circ}$ and $120^{\circ}$, and we estimate a 5-sigma fluence upper limit (UL) of about $\left(2.0 \times 10^{-6}\right) \mathrm{erg} \mathrm{cm}^{-2}$ in the $450-10000 \mathrm{keV}$ energy range, for a 1 -second exposure. 


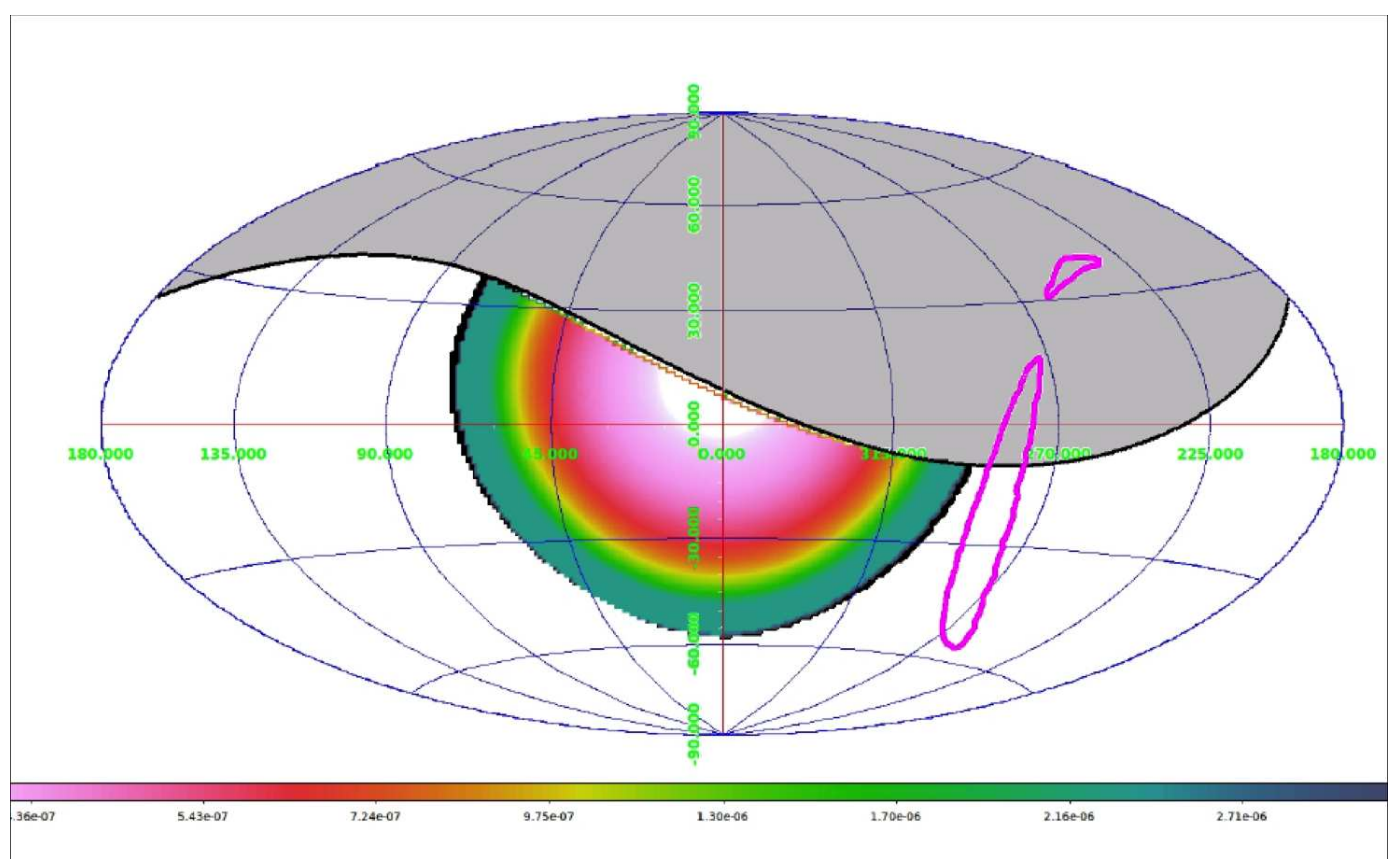

Figure 2: Hammer-Aitoff projection in Galactic coordinates. AGILE-GRID gamma-ray exposure (in $\mathrm{cm}^{2} \mathrm{~s}$ sr) during the 4-seconds interval that includes the GW150914 event time: AGILE just missed covering the error box at T0 with its GRID instrument.

\subsection{Search for possible precursor and delayed emission}

Even though GW150914 is a recognised BH-BH coalescence event, and current theoretical models in this case do not envision gamma-ray emission, a residual gaseous and/or plasma environment can in principle induce e.m. radiation during the approaching phase through non-thermal processes. It is then of great interest to explore this possibility and search for e.m. counterparts also for GW events resulting from the coalescence of two BHs, as in the case of GW150914. We carried out a search for a gamma-ray precursor and delayed emission over a large dynamic time range, up to several days after the event. The complete results of the individual passes analysis were reported in [5]. One of the most interesting passes is the first useful AGILE-GRID pass after the T0, $\Delta T_{+1}$, which covers the time interval $T 0+250<T<T 0+350 \mathrm{~s}$. The gamma-ray $2 \sigma \mathrm{UL}$ in this case is $\mathrm{UL}=1.9 \times 10^{-8} \mathrm{erg} \mathrm{cm}^{-2} \mathrm{~s}^{-1}$ in the range $50 \mathrm{MeV}-10 \mathrm{GeV}$.

The short GRB 090510 gamma-ray light curve $[11,10]$ shows several features expected from compact object coalescences, such as a soft precursor occurring $\sim 0.5 \mathrm{~s}$ before the main hard $\mathrm{X}$-ray event, a very luminous prompt and delayed gamma-ray afterglow emissions, and it was used as a tentative high-energy template counterpart also for the BH-BH GW150914 event [9]. In Figure 3 the AGILE-GRID (blue circles) [10] and Fermi-LAT (black squares) [11] gamma-ray light curves of the short GRB 090510 (originally at $\mathrm{z}=0.9$ ) were time-corrected and scaled in flux at a luminosity distance of $400 \mathrm{Mpc}(\mathrm{z}=0.09)$ for GW150914. The AGILE upper limit to gamma-ray emission above $100 \mathrm{MeV}$ from the $65 \%$ of the GW150914 localization region during the time interval $\Delta T_{+1}$, obtained only about 250 seconds after T0 is marked in red [5]. Fermi-LAT could provide an upper limit for GW150914 more than one hour after the event, and it is marked in black [9]. As shown in Figure 3, AGILE-GRID provided the most stringent constraint to any 


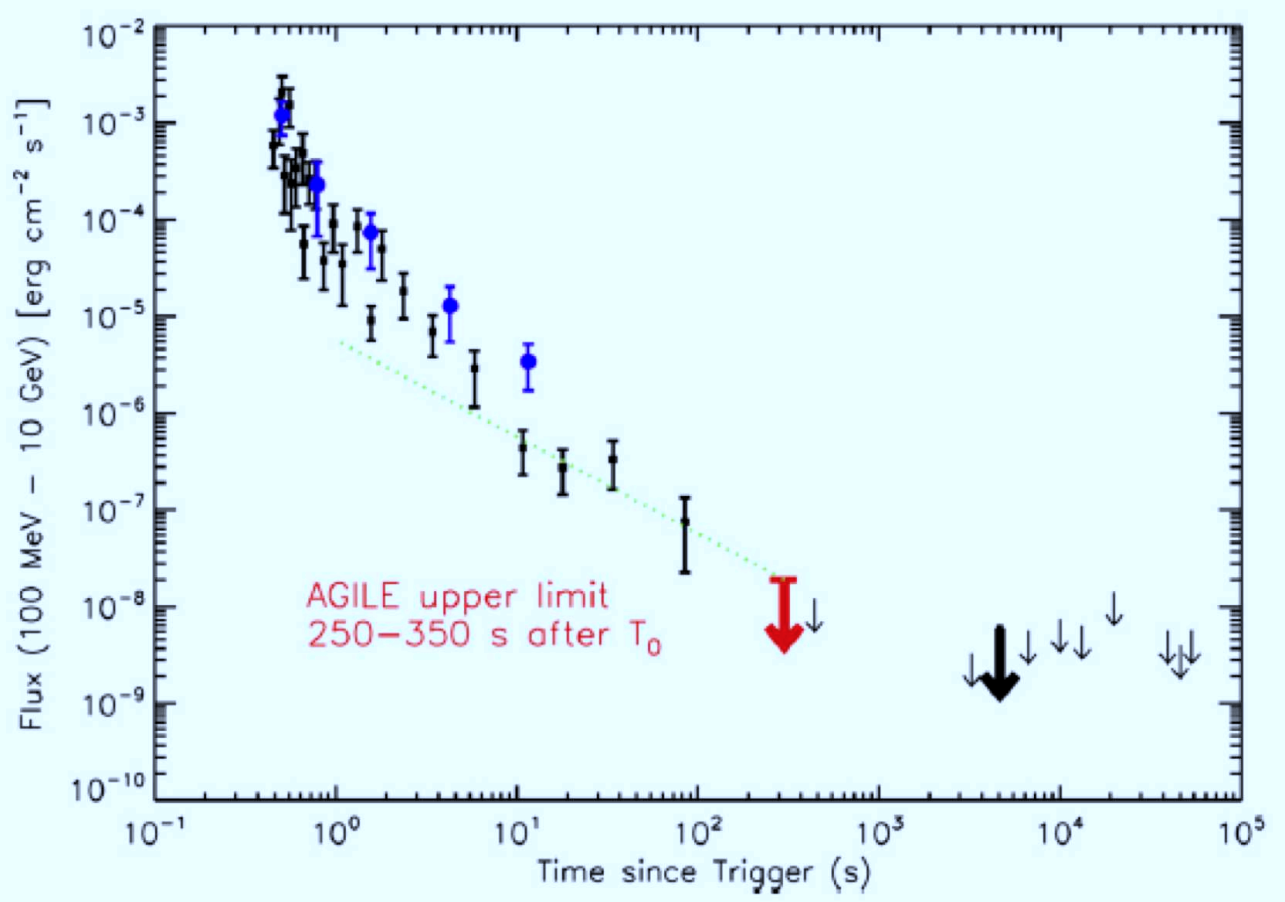

Figure 3: The short GRB 090510 light curve rescaled at $\mathrm{z}=0.09$ and used as a possible high-energy template counterpart for the GW150914 event [9]. The AGILE-GRID upper limit to gamma-ray emission above 100 $\mathrm{MeV}$ is shown in red and it is the the most stringent constraint to any delayed emission above $100 \mathrm{MeV}$ after the GW150914 event [5]. The Fermi-LAT upper limit obtained in the interval $4442-4867 \mathrm{~s}$ after the event is marked in black [9].

delayed emission above $100 \mathrm{MeV}$ only about 250 seconds after the GW150914 event.

Our data are close to excluding a delayed gamma-ray afterglow for the GW150914 event of the same type as GRB 090510.

\section{AGILE and GW astrophysics}

AGILE can effectively observe relatively large sky regions associated with GW events with high efficiency. As demonstrated in the case of GW150914, AGILE might obtain significant results if the event occurs in the accessible sky region, which depends on the sky coverage per 7 minute rotation $(\sim 0.8)$ and Earth occultation $(\sim 0.6)$, which results in a probability of $\sim 50 \%$.

The very fast AGILE ground segment alert system [7] [8] has been recently further optimized for the search of electromagnetic counterparts of gravitational waves, allowing the AGILE Team to perform full GRID data reduction and the preliminary Quick Look scientific analysis only 25/30 minutes after the telemetry download from the spacecraft. The AGILE observations are significant in providing the fastest response to GW events with optimal gamma-ray sensitivity.

The AGILE Team is now part of the multifrequency follow-up collaboration with the LIGOVIRGO Collaboration, and further AGILE analysis of GW candidate events is in progress [12]. 
The prospects for future follow-up gamma-ray observations of GW sources by AGILE are very promising.

\section{References}

[1] Tavani, M. et al., A.\&A. 502, (2009) 995

[2] Abbott, B. P., Abbott, R., Abbott, T. D., et al. PhRvL 116 (2016)

[3] Veitch, J., Raymond, V., Farr, B., et al. 2015, PhRvD, 91, 042003

[4] Abbott, B. P., Abbott, R., Abbott, T. D., et al., ApJL 826 (2016)

[5] Tavani, M. et al., ApJL 825:L4 (2016)

[6] Connaughton, V. et al., ApJL 826:L6 (2016)

[7] Pittori, C., Nucl. Phys. B Proc. Suppl., 239 (2013)

[8] Bulgarelli, A. et al., ApJ 781:19 (2014)

[9] Ackermann, M. et al., 2016, ApJL, 823, L2

[10] Giuliani, A. et al. 2010, ApJL, 708, L84

[11] Ackermann, M. et al. 2010, ApJ, 716, 1178

[12] Verrecchia, F. et al. (2017), in progress 